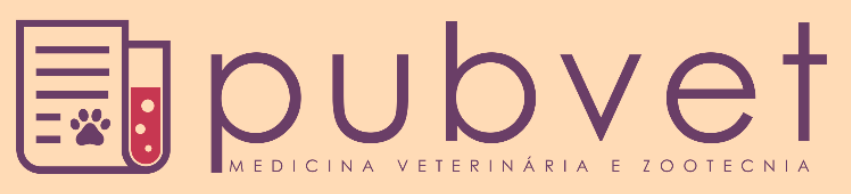

https://doi.org/10.31533/pubvet.v15n03a775.1-8

\title{
Utilização da hemilaminectomia nos casos de compressão medular por extrusão de disco intervertebral em cães: Revisão
}

\author{
Jennifer Santos ${ }^{*}$, Alisson Andrade ${ }^{1}$, Bibiana Freitas ${ }^{1}$, Rodrigo Otávio Cardona ${ }^{2} \bullet$ \\ ${ }^{I}$ Graduandos em Medicina Veterinária da Universidade de Cruz Alta. Cruz Alta- RS, Brasil. \\ ${ }^{2}$ Docente da Universidade de Cruz Alta. Cruz Alta- RS, Brasil. \\ *Autor para correspondência, E-mail: jenni.santos441@gmail.com
}

Resumo. A neurologia é a especialidade que cresce cada vez mais dentro da medicina veterinária, visto que as afecções neurológicas são comumente encontradas na clínica de pequenos animais. No entanto, muitas doenças neurológicas podem ser tratadas de maneira significativa, principalmente quando o diagnóstico é realizado precocemente, e o tratamento logo iniciado, podendo ser clínico ou cirúrgico. Uma das afecções mais comuns que acometem o sistema nervoso central é a doença do disco intervertebral (Hansen tipo I), caracterizada pela extrusão do disco intervertebral, provocando alterações neurológicas em cães, principalmente nos cães de pequeno porte. Nesse caso, quando o tratamento é cirúrgico, há várias abordagens cirúrgicas relatadas na literatura, sendo a hemilaminectomia a técnica cirúrgica mais comumente utilizada para a descompressão da medula espinhal. Dessa forma, por ser uma doença de alta severidade, este artigo tem como objetivo relatar as principais características desta enfermidade e o uso da técnica de hemilaminectomia para seu tratamento, no qual o prognóstico depende do grau de lesão neurológico, associado ao pós-operatório.

Palavras-chave: Cão, extrusão, medula espinhal, neurologia

\section{Use of hemilaminectomy in cases of spinal compression by extrusion of intervertebral disc in dogs: Review}

\begin{abstract}
Neurology is a specialty that grows more and more in veterinary medicine, seen as neurological disorders commonly found in the clinic of small animals. However, many neurological diseases can be treated significantly. Especially when the diagnosis is made early, and the logo of the treatment started, can be clinical or surgical. One of the most common diseases that affects the central nervous system is intervertebral disc disease (Hansen type I), characterized by the extrusion of the intervertebral disc, causing neurological changes in dogs, especially in small dogs. In this case, when the treatment is surgical, there are several surgical approaches related to the literature, with a hemilaminectomy being a surgical technique most commonly used for spinal cord decompression. Thus, since it is a highly serious disease, this article aims to list the main resources of this disease and the use of the hemilaminectomy technique for its treatment, without qualifying or prognosis, depends on the degree of neurological injury associated with the postoperative period.
\end{abstract}

Keywords: Dog, extrusion, spinal cord, neurology

\section{Uso de hemilaminectomía en casos de compresión espinal por extrusión de disco intervertebral en perros: Revisión}


Resumen. La neurología es una especialidad que crece cada vez más en la medicina veterinaria, en vista a que los trastornos neurológicos se encuentran comúnmente en la clínica de pequeños animales. Sin embargo, muchas enfermedades neurológicas pueden tratarse significativamente. Especialmente cuando el diagnóstico se realiza temprano, y el tratamiento es iniciado inmediatamente, pudiendo ser clínico o quirúrgico. Uno de los trastornos más comunes que afecta el sistema nervioso central es la enfermedad del disco intervertebral (Hansen tipo I), caracterizada por la extrusión del disco intervertebral, provocando cambios neurológicos en los perros, especialmente en perros pequeño. En este caso, cuando el tratamiento es quirúrgico, existen varios enfoques quirúrgicos reportados en la literatura, siendo la hemilaminectomía la técnica quirúrgica más utilizada para la descompresión medular. Así, por tratarse de una enfermedad de alta gravedad, este artículo tiene como objetivo dar a conocer las principales características de esta enfermedad y el uso de la técnica de hemilaminectomía para su tratamiento, en la que el pronóstico depende del grado de lesión neurológica, asociada al postoperatorio.

Palabras clave: Perro, extrusión, médula espinal, neurología

\section{Introdução}

Os discos intervertebrais são estruturas de conteúdo gelatinoso com a finalidade de conectar as vértebras, conferir rigidez e flexibilidade à coluna vertebral, além de absorver impactos. Estão localizados entre os corpos vertebrais de todas as vértebras, com exceção apenas de C1-C2 (atlas e áxis) e das vértebras sacrais (Bray \& Burbidge, 1998; Costa, 2001; Sharp et al., 2005).

Anatomicamente, os discos intervertebrais são compostos pelo anel fibroso e núcleo pulposo (Figura 1). O núcleo pulposo, corresponde a uma estrutura ovoide e central composta por material gelatinoso, e que se torna progressivamente desidratado e menos parecido com gel conforme a idade, sendo localizado internamente. Já o anel fibroso, é formado por material fibrocartilaginoso, onde os feixes colágenos são dispostos em camadas concêntricas. Essa estrutura é mais delgada na região dorsal, tornando mais fácil sua ruptura nessa região (Brown, 1977; Maggs et al., 2017; Sharp et al., 2005). Adjacentes aos discos encontram-se, os ligamentos longitudinais dorsais, ventrais e interceptais, cuja finalidade fundamenta-se na sustentação ao longo de toda coluna vertebral (Fossum, 2014; Sharp \& Wheeler, 2005; Slatter, 2007).

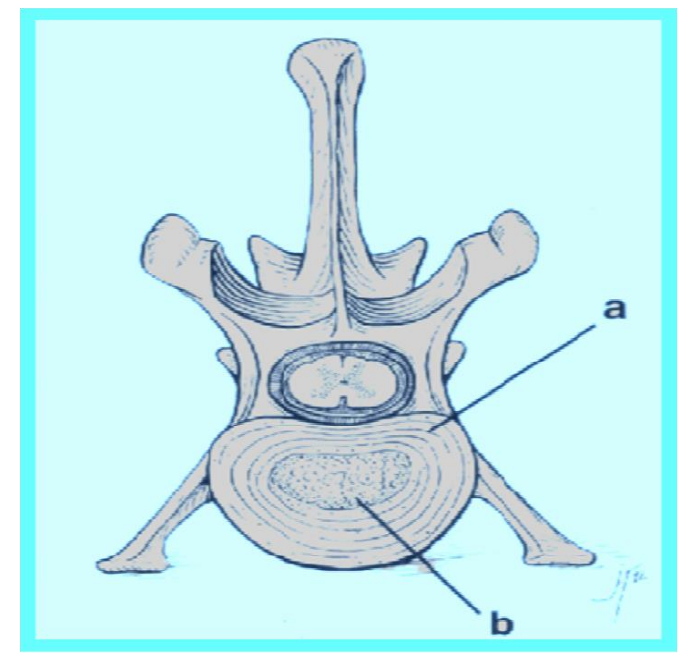

Figura 1. Doença do disco toracolumbar no cão: Uma análise restrocpectiva de 187 casos. Fonte: Brown et al. (1977)

Entretanto, fatores como predisposição genética, trauma automobilísticos, projéteis (lesões por armas de fogo), quedas e lesões provocadas por outros animais ou por objetos e envelhecimento, são causas que estabelecem um processo degenerativo no disco intervertebral (Brisson, 2010). Uma vez que essa degeneração no disco se intensifica ou ocorre precocemente, desencadeia uma série de alterações nos componentes adjacentes, principalmente em ligamentos, articulações e corpos vertebrais, o que resulta 
consequentemente em uma falha estrutural ao longo da coluna vertebral (Bergknut et al., 2013; Brisson, 2010; Hansen, 1952).

Dessa forma, afecções neurológicas são frequentemente descritas em cães, sendo a mais comum a doença do disco intervertebral (DDIV), apesar de ser mais frequente em animais de raças condrodistróficas, mesmo que raramente, pode acometer cães de raça não condrodistrófica. Esta enfermidade, caracteriza-se por degeneração do disco intervertebral, resultando em extrusão ou protrusão do conteúdo discal em direção ao canal vertebral (Hansen, 1952). No que se refere, a degeneração do disco intervertebral com ruptura do anel fibroso e extrusão do núcleo pulposo, esta foi caracterizada como causa de compressão da medula espinhal desde 1930 (Hansen, 1952). Além de ser considerada a causa mais comum de lesão severa da medula espinhal em cães e estima-se que acometa em torno de $1 \%$ de todos os casos vistos na clínica de pequenos animais (Olby, 2010).

\section{Desenvolvimento}

A doença do disco intervertebral (DDIV) é uma afecção provocada pela degeneração do disco intervertebral. Pode ocorrer a extrusão (Hansen tipo I) ou protrusão (Hansen tipo II) do disco para o interior do canal vertebral. Tanto na doença de Hansen tipo 1 quanto na de Hansen tipo 2 ocorre compressão da medula espinhal (Risio et al., 2014; Sharp \& Wheeler, 2005). A extrusão do disco intervertebral (EDIV) ou Hansen tipo I geralmente acontece quando o disco intervertebral sofre degeneração condroide e envolve a migração hiperaguda do material do núcleo pulposo através de todas as camadas do anel fibroso rompido para o interior do canal vertebral (Figura 2) (Jeffery, 1995).

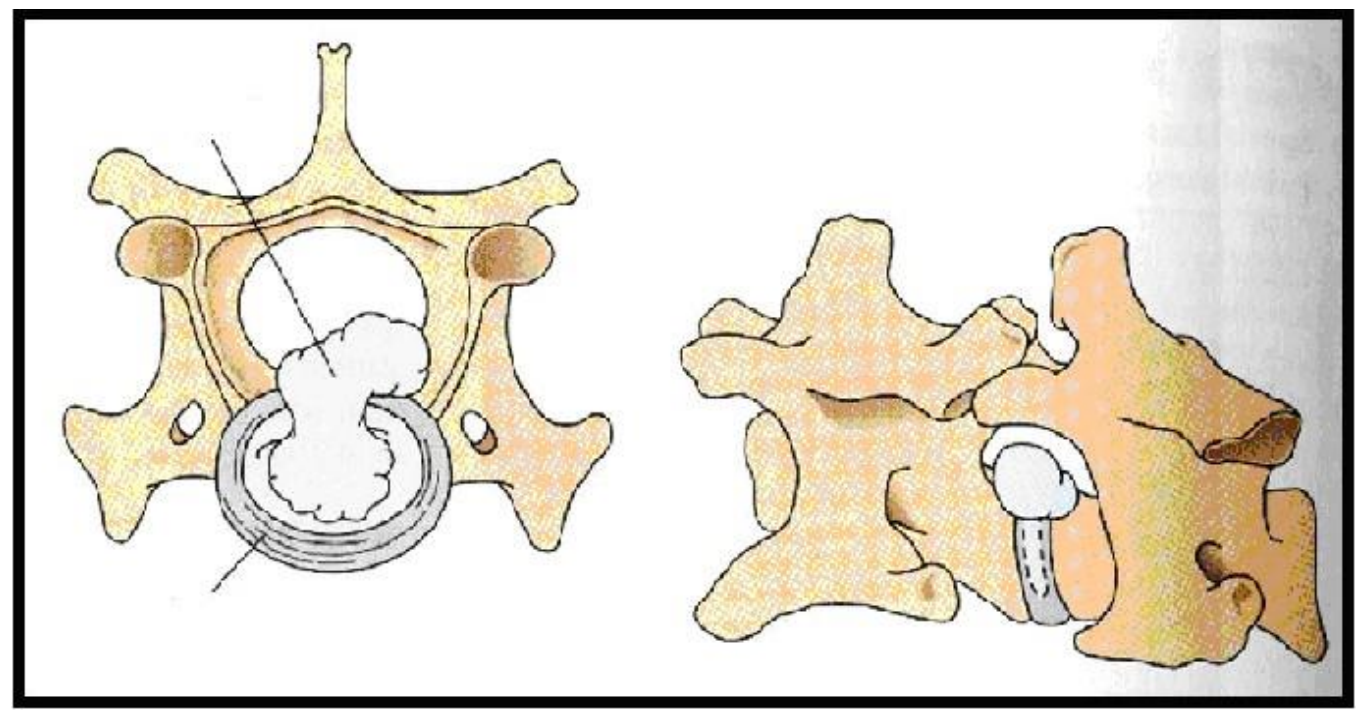

Figura 2. Lesão de Hansen tipo 1. Pode-se observar material degenerado dentro do canal vertebral. Fonte: Fossum (2014)

As forças anormais geradas pela degeneração e mineralização do núcleo pulposo levam ao desenvolvimento de ruptura do anel fibroso, com quebra de cada camada formando um túnel do qual o núcleo pulposo anormal pode eventualmente deslocar-se para o interior do canal medular (Brisson, 2010; De Lahunta et al., 2014; Hansen, 1952) ocasionando os sinais clínicos devido à compressão medular focal e em alguns casos da raiz do nervo espinhal (Toombs \& Waters, 2007). O material nuclear pode estar disperso por um segmento grande da medula espinhal, ou localizado, em estreita proximidade com o espaço ocupado pelo disco afetado. A ruptura pode ser lateral ao ligamento longitudinal dorsal, podendo ocorrer uma extrusão de forma irregular, plana, em padrão circular ou cônico. O material do disco que sofreu extrusão é irregular, quebradiço, granulado, às vezes parece gesso e varia de brancoamarelo para cinza-amarelo ou mesmo cinza-vermelho se houver a presença de sangue misturado, devido à consequente lesão do seio venoso na DDIV em cães (Brisson, 2010). Quando esta extrusão se torna crônica, em geral por não ter sido realizado o tratamento cirúrgico, o material do núcleo pulposo fibrinoso pode aderir-se à dura-máter ou pode ser reabsorvido (Brisson, 2010). 
Na EDIV o núcleo pulposo desidrata, as células degeneram e toda a estrutura se torna calcificada, o que altera a distribuição de pressão intradiscal, causando focos de estresse mecânico no anel fibroso. Com o tempo, podem ocorrer rupturas nos filamentos de colágeno até uma falha mecânica final possibilitando assim, a extrusão do conteúdo pulposo em direção ao canal medular (Jeffery, 1995). Este tipo de herniação tem evolução aguda e ocorre geralmente na coluna toracolombar e cervical (Bergknut et al., 2013; Smolders et al., 2013).

Em síntese, as extrusões de Hansen tipo I ocorrem com grande frequência em cães de pequeno porte, principalmente em raças condrodistróficas como o Dachshund, Lhasa Apso, Shih Tzu, Pequinês, Beagle e Basset Hound, cuja idade predominante encontra-se entre três e seis anos (Dewey, 2014; Kerwin et al., 2012; Taylor, 2015). A degeneração e a calcificação precoce do disco intervertebral estão associadas com genes recentemente identificados em animais condrodistróficos, sugerindo a existência de uma etiologia multigenética (Smolders et al., 2013). A EDIV geralmente ocorre de forma isolada, sendo incomum acometer dois ou mais locais concomitantes (Aikawa, 2012; Aikawa et al., 2012; Brisson, 2010; Chaves et al., 2015).

As manifestações clínicas dependem da localização e da gravidade da lesão, do volume de material no interior do canal vertebral e da velocidade com que esse material é ejetado. Pacientes acometidos por Hansen tipo I comumente apresentam alterações clínicas de forma aguda com evolução de minutos a dias (Fingeroth \& Thomas, 2015).

O principal sinal clínico da doença é a dor (Costa, 2001). Outros sinais são: hiperestesia espinhal, caracterizada por cifose e relutância em caminhar, perda parcial da função sensorial e motora voluntária, caracterizada por ataxia e paraparesia, progredindo para paraplegia com ou sem incontinência urinária e fecal e paraplegia sem perda da dor profunda (nocicepção) (Brisson, 2010; Fingeroth \& Thomas, 2015; Granger \& Carwardine, 2014).

O diagnóstico da doença pode ser feito com base no histórico do paciente, sua predisposição genética para a ocorrência da enfermidade, realização de exames físicos e neurológicos e os achados de exames complementares como radiografias simples, mielografia, tomografia computadorizada e a ressonância magnética (Almeida et al., 2018; Penning et al., 2006; Tamura et al., 2015; Tanaka et al., 2004).

Quanto ao tratamento, este pode ser clinico ou cirúrgico, porém deve-se levar em consideração o grau de disfunção neurológica (Brisson, 2010; Fingeroth \& Thomas, 2015). O tratamento clínico geralmente é indicado para cães com hiperestesia espinhal associada ou não a mínimas deficiências neurológicas (graus I e II) e se baseia no uso de anti-inflamatórios esteroidais ou não esteroidais, analgésicos, relaxantes musculares e a restrição de movimentos durante duas a quatro semanas (Bojrab, 2005; Santos et al., 2011; Sharp \& Wheeler, 2005; Taylor, 2015). Frequentemente na terapia médica da DDIV são empregados glicocorticoides com objetivo de reduzir edema, estresse oxidativo e resposta inflamatória perimedular, no entanto, a real eficácia da utilização dessa classe medicamentosa ainda não foi comprovada (Brisson, 2010).

Já o tratamento cirúrgico, é indicado quando não há resposta ao tratamento clínico, sinais clínicos redicivantes ou progressivos, paraparesia não ambulatória (grau III), paraplegia com presença de dor profunda (grau IV) ou ausência da dor profunda (grau V) com duração inferior a 48 horas (Bojrab, 2014). Quando há perda da sensibilidade profunda e o tratamento cirúrgico é feito antes das $48 \mathrm{~h}$, a chance de o animal retornar as suas funções é de 25 a $76 \%$, porém quando realizada após as $48 \mathrm{~h}$, esta chance diminui para 5\% (Arias et al., 2007).

Salienta-se, que antes dos procedimentos cirúrgicos são administrados corticosteroides intravenosos para que a medula espinhal fique protegida contra os efeitos da manipulação cirúrgica. Recomenda-se o uso de Succinato Sódico de Metilprednisolona ( $30 \mathrm{mg} / \mathrm{kg}$ ), ao invés de Dexametasona, pois esta última substância causa vários efeitos colaterais (Toombs \& Bauer, 1998).

Existem várias técnicas cirúrgicas para a descompressão da medula espinhal por extrusão de disco intervertebral, como hemilaminectomia, pediculectomia, minihemilaminectomia, laminectomia dorsal e corpectomia lateral, consideradas as mais utilizadas (Brisson, 2010; Kerwin et al., 2012; Sharp \& Wheeler, 2005). Além disso, descreve-se a técnica de fenestração discal como método profilático para 
futuras herniações, no entanto, a realização deste procedimento sempre deve ser adjunta a alguma outra técnica corretiva e nunca de forma isolada (Bojrab, 2005; Dewey, 2014; Mazantti et al., 2013).

$\mathrm{Na}$ doença do disco intervertevbral (Hansen tipo I), a finalidade do tratamento cirúrgico é a descompressão da medula espinhal para remoção do material do disco extrusado, eliminação da pressão sobre a medula espinhal e restauração da perfusão tecidual normal (Arias et al., 2007).

Entre as técnicas descompressivas, a hemilaminectomia é o procedimento cirúrgico mais indicado para casos de compressão medular por extrusão (Kerwin et al., 2012). Esta técnica cirúrgica fundamentase na excisão unilateral da lâmina óssea, dos processos articulares cranial e caudal e de partes do pedículo das vértebras envolvidas. Por ser um procedimento que causa menos instabilidade vertebral, é preferível em relação à laminectomia, pois preserva a integridade estrutural e mecânica da coluna vertebral, é menos traumática, é mais cosmética, reduz a chance de formação de fibrose epidural que causa compressão da medula espinhal (Fossum, 2014), e foi observado que os cães apresentam significante melhora na recuperação da função neurológica (Muir et al., 1995).

Além disso, permite uma descompressão satisfatória da medula espinhal, uma vez que o acesso cirúrgico é realizado diretamente na porção ventral do canal intervertebral, local este onde ocorre a maioria das lesões compressivas (Bojrab, 2005; Dewey, 2014; Kerwin et al., 2012). Todavia, apresenta um risco aumentado de hemorragia do seio venoso em comparação com o procedimento de laminectomia dorsal (Brisson, 2010). Entretanto, para a execução da técnica se faz necessário à localização exata da área comprimida, uma vez que o acesso cirúrgico dificilmente permite a remoção contralateral de materiais extrusados sem excessiva manipulação da medula espinhal (Bojrab, 2005; Mazantti et al., 2013; Toombs \& Waters, 2007).

O prognóstico depende de fatores como a duração dos sinais clínicos (Kazakos et al., 2005; $\underline{\text { Scott \& }}$ McKee, 1999) e qualidade dos cuidados realizados no período pós-operatório (Jerram et al., 1997). A classificação da DDIV em graus facilita a escolha do tratamento, garantindo melhores resultados. A técnica cirúrgica de hemilaminectomia, com a retirada do material do disco do interior do canal vertebral é uma forma efetiva de tratamento. Alguns estudos relatam resultados satisfatórios do tratamento cirúrgico em cães com DDIV toracolombar com diferentes graus de disfunção neurológica (Aikawa, et al., 2012; Ingram et al., 2013; Kazakos et al., 2005; Kranenburg et al., 2013). Além disso, pacientes diagnosticados com Hansen tipo I com presença de nocicepção e quando submetidos à descompressão cirúrgica recuperam a função neuro motora acima de $80 \%$ em aproximadamente duas semanas pós operatório (Dewey, 2014).

Convém ressaltar, que terapias adjuvantes associadas ao tratamento cirúrgico de pacientes com DDIV são frequentemente recomendadas, como por exemplo, fisioterapia passiva e acupuntura e contribuem para um prognóstico favorável (Taylor, 2015).

\section{Considerações finais}

As doenças compressivas da medula espinhal em cães têm sido relatadas com frequência na clínica de pequenos animais, no qual a raça e a idade do animal são fatores importantes a serem observados, pois raças condrodistróficas entre três e sete anos são muito predisponentes a desenvolverem DDIV, porém não se pode descartar a hipótese de que outras raças possam ser afetadas. Assim, na doença do disco intervertebral do tipo I, a descompressão medular por meio da técnica de hemilaminectomia, para remoção de material do disco extrusado, tem sido considerada a cirurgia mais eficaz, devido inúmeras vantagens que apresenta, pois o prognóstico favorável depende além do diagnóstico correto e precoce, da intervenção cirúrgica para descompressão medular, visto que uma técnica cirúrgica adequada oferece ao paciente maiores chances de recuperação de suas funções neuromotoras e consequentemente melhor qualidade de vida.

\section{Referências}

Aikawa, T., Fujita, H., Kanazono, S., Shibata, M., \& Yoshigae, Y. (2012). Long-term neurologic outcome of hemilaminectomy and disk fenestration for treatment of dogs with thoracolumbar intervertebral disk herniation: 831 cases (2000-2007). Journal of the American Veterinary Medical Association, 241(12), 1617-1626. DOI: https://doi.org/10.2460/javma.241.12.1617 
Aikawa, T., Fujita, H., Shibata, M., \& Takahashi, T. (2012). Recurrent thoracolumbar intervertebral disc extrusion after hemilaminectomy and concomitant prophylactic fenestration in 662 chondrodystrophic dogs. Veterinary Surgery, 41(3), 381-390. DOI: https://doi.org/10.1111/j.1532950x.2012.00970.x

Almeida, J. A. N. C., Medeiros, T. T. B., Carreiro, A. N., Cunha, E. M., Araújo, D. V. F., Falcão, B. M. R., La Salles, A. Y. F., \& Menezes, D. J. A. (2018). Diagnóstico por tomografia computadorizada da extrusão de disco intervertebral em paciente geriatra: Relato de Caso. PUBVET, 12(3), 1-5. DOI: https://doi.org/10.22256/pubvet.v12n3a45.1-5

Arias, M. V. B., Nishioka, C. M., Garcia, C. O., Reia, A. Z., Júnior, D. B., \& Marcasso, R. A. (2007). Avaliação dos resultados clínicos após cirurgia descompressiva em cães com doença de disco intervertebral Evaluation of clinical results of decompressive surgery in dogs with degenerative disk disease. Arquivo Brasileiro de Medicina Veterinária e Zootecnia, 59(6), 1445-1450. DOI: https://doi.org/10.1590/s0102-09352007000600015

Bergknut, N., Smolders, L. A., Grinwis, G. C. M., Hagman, R., Lagerstedt, A. S., Hazewinkel, H. A. W., Tryfonidou, M. A., \& Meij, B. P. (2013). Intervertebral disc degeneration in the dog. Part 1: Anatomy and physiology of the intervertebral disc and characteristics of intervertebral disc degeneration. The Veterinary Journal, 195(3), 282-291. DOI: https://doi.org/10.1016/j.tvjl.2012.10.024

Bojrab, M J. (2005). Técnicas atuais em cirurgia de pequenos animais. Editora Roca.

Bojrab, M Joseph. (2014). Mecanismos da moléstia na cirurgia dos pequenos animais. Roca, Brasil.

Bray, J. P., \& Burbidge, H. M. (1998). The canine intervertebral disk: part one: structure and function. Journal of the American Animal Hospital Association, 34(1), 55-63. DOI: https://doi.org/10.5326/15473317-34-1-55

Brisson, B. A. (2010). Intervertebral disc disease in dogs. The Veterinary Clinics of North America. Small Animal Practice, 40(5), 829-858.

Brown, N. O., Helphrey, M. L. \& Prata, R. G. (1977). Thoracolumbar disk disease in the dog. A retrospective analysis of 187 cases. Jornal Animal Hasp Association, 13, 665-672.

Chaves, R. O., Ferantil, J. P. S., Corrêa, L. F. D., Copat, B., Polidoro, D., Gorczak, R., Libardoni, R. N., \& Mazzanti, A. (2015). Extrusão de disco intervertebral multifocal em cão. Acta Scientiae Veterinariae, 43, $1-4$.

Costa, R. C. (2001). Disco intervertebral: bases para o diagnóstico e tratamento da doença. Nosso Clínico, 4, 20.

De Lahunta, A., Glass, E. N., \& Kent, M. (2014). Veterinary neuroanatomy and clinical neurology (Vol. $1)$. W.B. Saunders.

Dewey, C. W. (2014). Cirurgia da coluna cervical. In T. W. Fossum (Ed.), Cirurgia de pequenos animais (pp. 1467-1507).

Fingeroth, James, \& Thomas, W. (2015). Advances in intervertebral disc disease in dogs and cats. Wiley-Blackwell.

Fossum, T. W. (2014). Cirurgia de pequenos animais (4th ed., Vol. 1). Elsevier Brasil.

Granger, N., \& Carwardine, D. (2014). Acute spinal cord injury: tetraplegia and paraplegia in small animals. Veterinary Clinics: Small Animal Practice, 44(6), 1131-1156. DOI: 10.1016/j.cvsm.2014.07.013

Hansen, H.-J. (1952). A pathologic-anatomical study on disc degeneration in dog: With special reference to the so-called enchondrosis intervertebralis. Acta Orthopaedica Scandinavica, 23(Sup 11), 1-130. DOI: https://doi.org/10.3109/ort.1952.23.suppl-11.01

Ingram, E. A., Kale, D. C., \& Balfour, R. J. (2013). Hemilaminectomy for thoracolumbar Hansen Type I intervertebral disk disease in ambulatory dogs with or without neurologic deficits: 39 cases (20082010). Veterinary Surgery, 42(8), 924-931. DOI: https://doi.org/10.1111/j.1532-950x.2013.12061.x

Jeffery, N. D. (1995). Handbook of small animal spinal surgery. WB Saunders.

Jerram, R. M., Hart, R. C., \& Schulz, K. S. (1997). Postoperative management of the canine spinal 
surgery patient. I. The Compendium on Continuing Education for the Practicing Veterinarian, 19(2), $147-161$.

Kazakos, G., Polizopoulou, Z. S., Patsikas, M. N., Tsimopoulos, G., Roubies, N., \& Dessiris, A. (2005). Duration and severity of clinical signs as prognostic indicators in 30 dogs with thoracolumbar disk disease after surgical decompression. Journal of Veterinary Medicine Series A, 52(3), 147-152. DOI: https://doi.org/10.1111/j.1439-0442.2005.00698.x

Kerwin, S. C., Levine, J. M., \& Hicks, D. G. (2012). Thoracolumbar Spine. In K. M. Tobias \& S. A. Johnston (Eds.), Veterinary surgery: small animal (Issue V700 TOBv, pp. 449-475). Elselvier Saunders.

Kranenburg, H.-J. C., Grinwis, G. C. M., Bergknut, N., Gahrmann, N., Voorhout, G., Hazewinkel, H. A. W., \& Meij, B. P. (2013). Intervertebral disc disease in dogs-Part 2: Comparison of clinical, magnetic resonance imaging, and histological findings in 74 surgically treated dogs. The Veterinary Journal, 195(2), 164-171. DOI: https://doi.org/10.1016/j.tvj1.2012.06.001

Maggs, D., Miller, P., \& Ofri, R. (2017). Slatter's Fundamentals of Veterinary Ophthalmology E-Book. Elsevier Health Sciences.

Mazantti, A. B., Beckmann, D. V, \& Santos, R. P. (2013). Princípios da neurocirúrgia. In A. L. A. Oliveira (Ed.), Técnicas cirúrgicas em pequenos animais (pp. 734-788). Elsivier.

Muir, P., Johnson, K. A., Manley, P. A., \& Dueland, R. T. (1995). Comparison of hemilaminectomy and dorsal laminectomy for thoracolumbar intervertebral disc extrusion in dachshunds. Journal of Small Animal Practice, 36(8), 360-367. DOI: https://doi.org/10.1111/j.1748-5827.1995.tb02950.x

Olby, N. (2010). The pathogenesis and treatment of acute spinal cord injuries in dogs. Veterinary Clinics: Small Animal Practice, 40(5), 791-807.

Penning, V., Platt, S. R., Dennis, R., Cappello, R., \& Adams, V. (2006). Association of spinal cord compression seen on magnetic resonance imaging with clinical outcome in 67 dogs with thoracolumbar intervertebral disc extrusion. Journal of Small Animal Practice, 47(11), 644-650. DOI: https://doi.org/10.1111/j.1748-5827.2006.00252.x

Risio, L., Thomas, W. B., \& Fingeroth, J. M. (2014). Traumatic dis extrusions. In J. Fingeroth \& W. Thomas (Eds.), Advances in intervertebral disc disease in dogs and cats (Wiley-Blac, pp. 121-127). John Wiley \& Sons.

Santos, R. P., Mazzanti, A., Beckmann, D. V, Berté, L., Ripplinger, A., Neto, D. P., \& Baumhardt, R. (2011). Recuperação funcional em cães com doença do disco intervertebral toracolombar sem percepção à dor profunda: 37 casos (2002-2010). Pesquisa Veterinária Brasileira, 31(4), 345-349. DOI: https://doi.org/10.1590/s0100-736x2011000400012

Scott, H. W., \& McKee, W. M. (1999). Laminectomy for 34 dogs with thoracolumbar intervertebral disc disease and loss of deep pain perception. Journal of Small Animal Practice, 40(9), 417-422. DOI: https://doi.org/10.1111/j.1748-5827.1999.tb03114.X

Sharp, N. J. H., Thumpey, J. E., \& Wheeler, S. J. (2005). Trastornos vertebrales de pequeños animales: diagnóstico y cirugía. Elsevier Mosby.

Sharp, N. J., \& Wheeler, S. J. (2005). Small animal spinal disorders: diagnosis and surgery. Elsevier Mosby.

Slatter, D. H. (2007). Manual de cirurgia de pequenos animais. Manole.

Smolders, L. A., Bergknut, N., Grinwis, G. C. M., Hagman, R., Lagerstedt, A.-S., Hazewinkel, H. A. W., Tryfonidou, M. A., \& Meij, B. P. (2013). Intervertebral disc degeneration in the dog. Part 2: chondrodystrophic and non-chondrodystrophic breeds. The Veterinary Journal, 195(3), 292-299. DOI: https://doi.org/10.1016/j.tvj1.2012.10.011

Tamura, S., Doi, S., Tamura, Y., Takahashi, K., Enomoto, H., Ozawa, T., \& Uchida, K. (2015). Thoracolumbar intradural disc herniation in eight dogs: clinical, low-field magnetic resonance imaging, and computed tomographic myelography findings. Veterinary Radiology \& Ultrasound, 56(2), 160-167. DOI: https://doi.org/10.1111/vru.12213

Tanaka, H., Nakayama, M., \& Takase, K. (2004). Usefulness of myelography with multiple views in diagnosis of circumferential location of disc material in dogs with thoracolumbar intervertebral disc 
herniation. Journal of Veterinary Medical Science, 66(7), 827-833. DOI: https://doi.org/10.1292/jvms.66.827

Taylor, S. M. (2015). Distúrbios da Medula Espinhal. In R. W. Nelson \& C. G. Couto (Eds.), Medicina Interna de Pequenos Animais (pp. 1048-1072). Elsevier Brasil.

Toombs, J. P., \& Bauer, M. S. (1998). Afecção do disco intervertebra. In D. Slatter (Ed.), Manual de cirurgia de pequenos animais (pp. 1286-1305).

Toombs, J. P., \& Waters, D. J. (2007). Afecção do disco intervertebral. In D. Slatter (Ed.), Manual de cirurgia de pequenos animais (pp. 1193-1208). Manole.

\section{Histórico do artigo:}

Recebido: 4 de agosto de 2020.

Aprovado: 8 de setembro de 2020.

Disponível online: 19 de janeiro de 2021
Licenciamento: Este artigo é publicado na modalidade Acesso Aberto sob a licença Creative Commons Atribuição 4.0 (CC-BY 4.0), a qual permite uso irrestrito, distribuição, reprodução em qualquer meio, desde que o autor e a fonte sejam devidamente creditados 